\title{
Sky coverage with the auxiliary telescopes Laser Guide Star tilt recovery technique
}

\author{
E. Marchetti ${ }^{1}$ and R. Ragazzoni ${ }^{1,2}$ \\ 1 CISAS, vicolo dell'Osservatorio 5, I-35122 Padova, Italy \\ e-mail: marchetti@astrpd.pd.astro.it \\ 2 Astronomical Observatory of Padova, vicolo dell'Osservatorio 5, I-35122 Padova, Italy \\ e-mail: ragazzoni@astrpd.pd.astro.it
}

Received October 14, 1996; accepted January 21, 1997

\begin{abstract}
Auxiliary telescopes in the neighbourhoods of a main telescope equipped with an LGS can provide, in principle, full sky coverage. The auxiliary telescopes, however, should be able to move around in order to acquire a useful natural guide star (NGS), and to track the LGS, comoving with the scientific target. Detailed expressions and results of numerical simulations are given.
\end{abstract}

Key words: atmospheric effects - methods: observational - telescopes

\section{Introduction}

One of the most relevant problem given by the use of a Laser Guide Star (LGS) to correct wavefront distortions in Adaptive Optics systems is the indetermination of the absolute tilt (Pilkington 1987; Olivier et al. 1993). This problem arises from the fact that laser beam fired from the ground passes through the atmosphere and, under its influence, generates an LGS whose relative position with respect to the scientific target changes continously. The tilt component of the turbulence detected by the science telescope is strongly degraded by the additional tilt motion of the LGS. A number of tentative solutions to the LGS tilt determination problem have been proposed (Belen'kii 1994, 1995; Foy et al. 1992, 1995; Rigaut \& Gendron 1992; Ragazzoni 1996a,b; Ragazzoni \& Marchetti 1996; Lukin 1996). Moreover Ragazzoni et al. (1995, REM95 hereafter) have considered a system of two small moving telescopes in the neighbourhood of the main observatory which are able to measure the undesired LGS tilt observing it from different angles of view and comparing its motion with respect to a natural star that lies in the same isokinetic patch.

Send offprint requests to: E. Marchetti
It is to be recalled that other investigators considered the option to have some moving telescopes, especially for interferometric purposes (Vivekanand et al. 1988).

In this paper we further investigate the REM95 solution, especially concerning the ground area requested by the movements of the auxiliary telescopes in order to provide a full sky coverage.

The core of the paper is subdivided into three sections; i): the limiting magnitude reachable by the auxiliary telescope is derived and discussed; $i i)$ : the relationships useful to figure out the geometrical path to be followed by such small telescopes are worked out and briefly discussed; iii): simulations, especially using MonteCarlo techniques, in order to obtain the ground coverage for the auxiliary telescopes are described.

\section{Limiting magnitude for the auxiliary telescopes}

We assume that the observed astronomical target is highorder corrected with the LGS itself. In this way its size shrinks to a diffraction limited core. Following Olivier \& Gavel (1994), but using a circular entrance pupil of diameter $D$, the final resolution experienced in presence of a residual jitter $\sigma \mathrm{rms}$ exceeds the diffraction limit by the factor $F$ given from:

$F=\frac{\sqrt{(1.22 \lambda / D)^{2}+(\sigma / 0.42)^{2}}}{1.22 \lambda / D}$.

Accepting a degradation of the resolution about $30 \%$ more than the diffraction limit one can find from Eq. (1), using $F=1.3$, a maximum residual jitter of $\sigma \approx 0.42 \lambda / D$, a value comparable to other figures given in the literature (Olivier et al. 1993). Defined an aperture diameter of the auxiliary telescopes $D_{\mathrm{a}}$ and an integration time $\Delta t$ a single exposure will collect $N_{*}$ photons given, for an A0V star of mag $V$, by (Zombeck 1990):

$N_{*} \approx 10^{8} 10^{-0.4 V} \frac{\pi D_{\mathrm{a}}^{2}}{4} \Delta t \Delta \lambda q$ 
where $\Delta \lambda$ is expressed in $\mathrm{nm}$ and $q$ is the overall quantum efficiency. Assuming a Poissonian noise the $S N R$ of the observed star is just $\sqrt{N_{*}}$ and, following Tyler \& Fried (1982), one gets:

$\sigma \approx \frac{3 \pi}{16} \frac{1}{\sqrt{N_{*}}} \frac{\lambda}{r_{0}} \chi$.

The additional $\chi$ factor has been introduced by Olivier \& Gavel (1994) and it is related to the ratio between the sampling and the control loop frequency. When such a ratio is 10 as they suggest, some $\chi=0.52$ is obtained. Combining Eqs. (1)-(3) and assuming the auxiliary telescopes as two reflection mirrors and high Q.E. CCD, an overall $q=0.8$ can be figured out; a bandwidth $\Delta \lambda=300 \mathrm{~nm}$ and a sampling time $\Delta t=10^{-2} \mathrm{~s}$ are used as a baseline for the following computation. In this way one get the following relationship for the limiting magnitude $V$ :

$V \approx 21.4-5 \log \frac{D}{D_{\mathrm{a}} r_{0}}$

where the argument of the logarithm should be expressed in meters.

Using Bahcall \& Soneira (1981) it is easy to retrieve the area in the sky where the probability to get one star is close to $100 \%$.

However the right-handed parameters in Eq. (4) have to be fixed. In the rest of the paper we assume a Fried parameter of $r_{0}=0.15 \mathrm{~m}$ in the visible, corresponding to a median seeing of most of the telescope sites where large telescopes do exist or are planned in the near term. The ratio $D / D_{\text {a }}$ between the diameters of the main and auxiliary telescope is a more complicated issue. In fact the smaller is the ratio the smaller movements will be requested to the auxiliary telescope. On the other hand it is to be pointed out that it is easier to move around smaller telescopes rather than larger ones. Ratios smaller than the unity are, obviously, unefficents. We adopted in the following a figure of $D / D_{\mathrm{a}}=8$. This translates into very small and light, $D_{\mathrm{a}} \approx 0.5 \mathrm{~m}$, telescopes for a $D=4 \mathrm{~m}$ class telescope and into $D_{\mathrm{a}} \approx 1 \mathrm{~m}$ telescopes for $D=8 \mathrm{~m}$ large telescope class.

When $D / D_{\mathrm{a}}=8$ and $r_{0}=0.15 \mathrm{~m}$ a limiting magnitude $V=12.8$ is obtained equal to a density of $n \approx 36$ stars per square degree at the North Galactic Pole (the worst case) or, in other words, to a mean search area of $1 / n \approx 100$ squared arcmin for a single star.

However, as pointed out by REM95 two auxiliary observers are required, forming an angle $\psi \approx 90^{\circ}$ with respect to the main observatory.

In the most general case the angle $\psi$ between the two tilt reference stars with respect the target star will differ from the ideal situation of $90^{\circ}$ and the error associated to tilt measurement could increase. The amount of this effect can be easily calculated: one can align one axis $(x)$ with one reference star (see Fig. 1) measuring tilt error along it then one can retrieve the error relative to the perpendicular axis $(y)$ projecting onto it the tilt error of the second reference star measured on the oblique axis $\left(y^{\prime}\right)$.

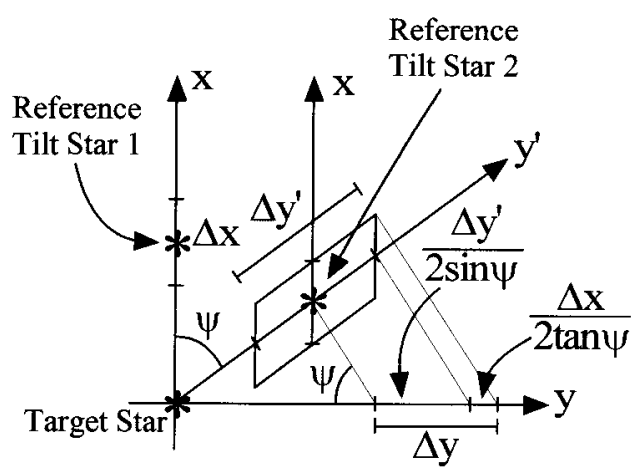

Fig. 1. The tilt error increasing is due to non-perpendicular configuration of auxiliary telescopes. The indetermination becomes larger as $\psi$ decreases

The error along the perpendicular axis $\Delta y$ will be given by:

$\Delta y=\sqrt{\left(\frac{\Delta y^{\prime}}{\sin \psi}\right)^{2}+\left(\frac{\Delta x}{\tan \psi}\right)^{2}}$.

Assuming the errors in determining $\Delta x$ and $\Delta y^{\prime}$ are the same, and equal to $\sigma$, an error $\sigma^{\prime}$ for the orthogonal axis $y$ can be worked out:

$\sigma^{\prime}=\sigma \sqrt{\frac{1+\cos ^{2} \psi}{\sin ^{2} \psi}}$

for departures from $\psi=90^{\circ}$ as much as $\pm 22.5^{\circ}$, an acceptable further degradation of $\approx 15 \%$ is obtained.

\section{Path of the auxiliary telescopes}

Because of Earth rotation it is necessary to move auxiliary telescopes around the main one in order to maintain the perspective superposition between LGS and tilt reference star.

The tracking path shape depends on several parameters: target star declination $\delta$, its angular separation $\theta$ from the tilt reference star and position angle PA of the reference with respect to the target. The geometry of the problem is shown in Fig. 2. In the following an height $H \approx 90 \mathrm{~km}$ (Happer et al. 1995) for the mesospheric Sodium layer is assumed.

To obtain tilt reference star position in the Sodium Layer plane coordinate system, placed at an altitude $H$ and centered on the LGS, two rotations are needed: the first around the $y^{\prime}$ axis by $90^{\circ}-h$ degrees, in order to 


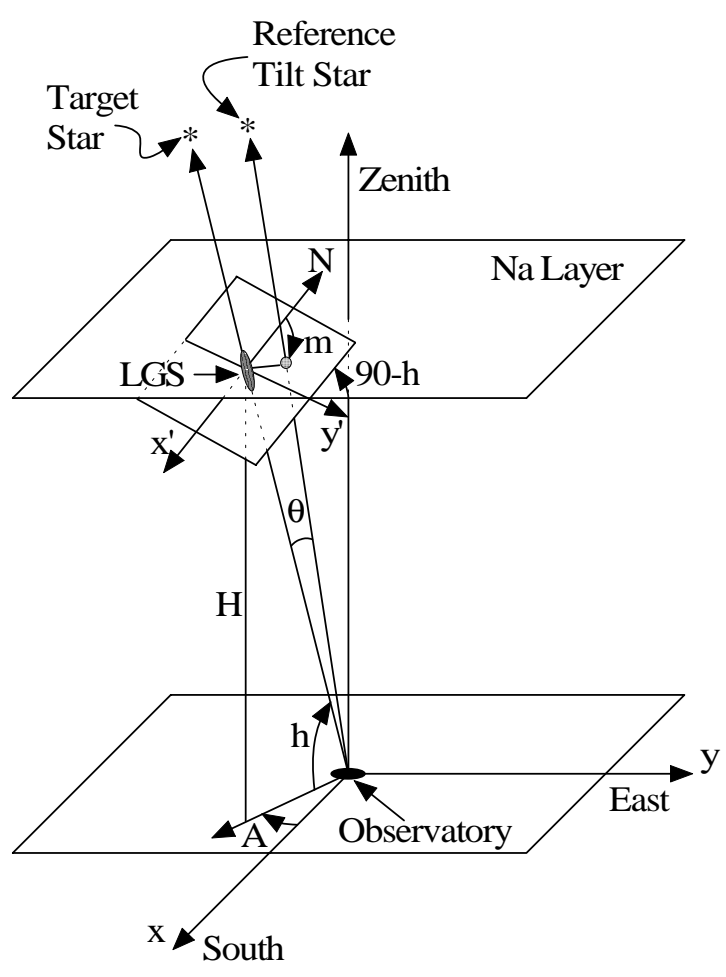

Fig. 2. Geometrical layout of the LGS and tilt reference star with respect to the observatory. The observatory plane coordinates are South-East oriented while the coordinates of the plane tangent to celestial sphere in the LGS are "altazimuthal-like" oriented with North along $x^{\prime}$ axis but with opposite versus

make parallel the plane tangent to the celestial sphere in the LGS and the Sodium Layer plane, the second around the axis passing through the LGS and perpendicular to the Sodium Layer plane, by the azimuth angle $A$, in order to align $x^{\prime} y^{\prime}$ axes to $x y$ axes:

$$
\left(\begin{array}{c}
\Delta x \\
\Delta y
\end{array}\right)=\left(\begin{array}{cc}
\cos A & \sin A \\
-\sin A & \cos A
\end{array}\right) \times\left(\begin{array}{ll}
\sin h & 1
\end{array}\right) \times\left(\begin{array}{c}
-\rho \cos m \\
\rho \sin m
\end{array}\right)(7)
$$

where the azimuth $A$, the height $h$ and the projected separation $\rho$ on the Sodium Layer plane are given by the following set of relationships:

$$
\left\{\begin{array}{l}
\tan A=\frac{\sin H A}{\cos H A \sin \phi-\tan \delta \cos \phi} \\
\sin h=\sin \phi \sin \delta+\cos \phi \cos \delta \cos H A \\
\rho=\frac{H}{\sin h} \theta
\end{array}\right.
$$

being $\phi$ the observatory latitude and $H A$ the target hour angle. The angle $m$ is related to the parallactic angle $q$ by the following pair of equations:

$$
\left\{\begin{array}{l}
m=\mathrm{PA}-q \\
\tan q=\frac{\sin H A}{\tan \phi \cos \delta-\sin \delta \cos H A}
\end{array} .\right.
$$

One can finally retrieve the auxiliary telescope position taking into account (see Fig. 3) that its coordinates will be identical to those of the tilt reference star on the Sodium Layer plane but with inverted sign:

$$
\left\{\begin{array}{c}
x=-\Delta x=\frac{H}{\sin h} \theta(\cos m \sin h \cos A-\sin m \sin A) \\
y=-\Delta y=-\frac{H}{\sin h} \theta(\cos m \sin h \sin A+\sin m \cos A) .
\end{array}\right.
$$

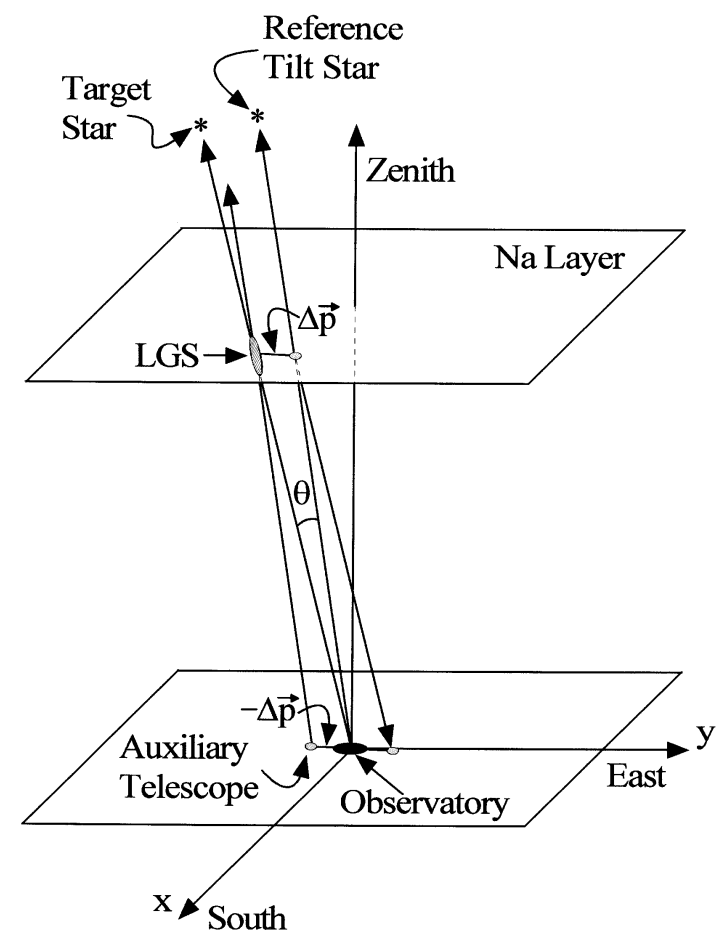

Fig. 3. Position of the auxiliary telescope with respect to observatory. Displacing the small telescope of an amount equal to the projection of the tilt reference star on Sodium Layer plane but with opposite versus, allows the auxiliary telescope to see LGS superimposed on the reference star

Some results obtained for the observatory latitude $\phi=$ $29^{\circ} \mathrm{N}$ (corresponding to the site of the National Telescope Galileo, Barbieri 1996) are shown in Fig. 4. The tracking is performed along four hours of $H A$ across meridian $\left( \pm 2^{\mathrm{h}}\right)$ and the filled circles are placed at steps of $30^{\mathrm{m}}$.

In Fig. 4a are drawn different paths calculated for values of $\delta$ interested by a zenithal distance of $45^{\circ}$ with a $5^{\circ}$ step, $\mathrm{PA}=-45^{\circ}$ and $\theta=0^{\circ} .2$; in this particular case the telescope movement is southward.

In Fig. 4b are shown paths with fixed $\mathrm{PA}=-45^{\circ}$ but different values of $\theta$ : note that, as expected, the shapes are identical but for a scale factor. In Fig. 4c the paths are calculated for different PA values and fixed $\theta=0.2$. The isolated filled dot identifies observatory position (located, in all cases, at the origin of the coordinate axis). 

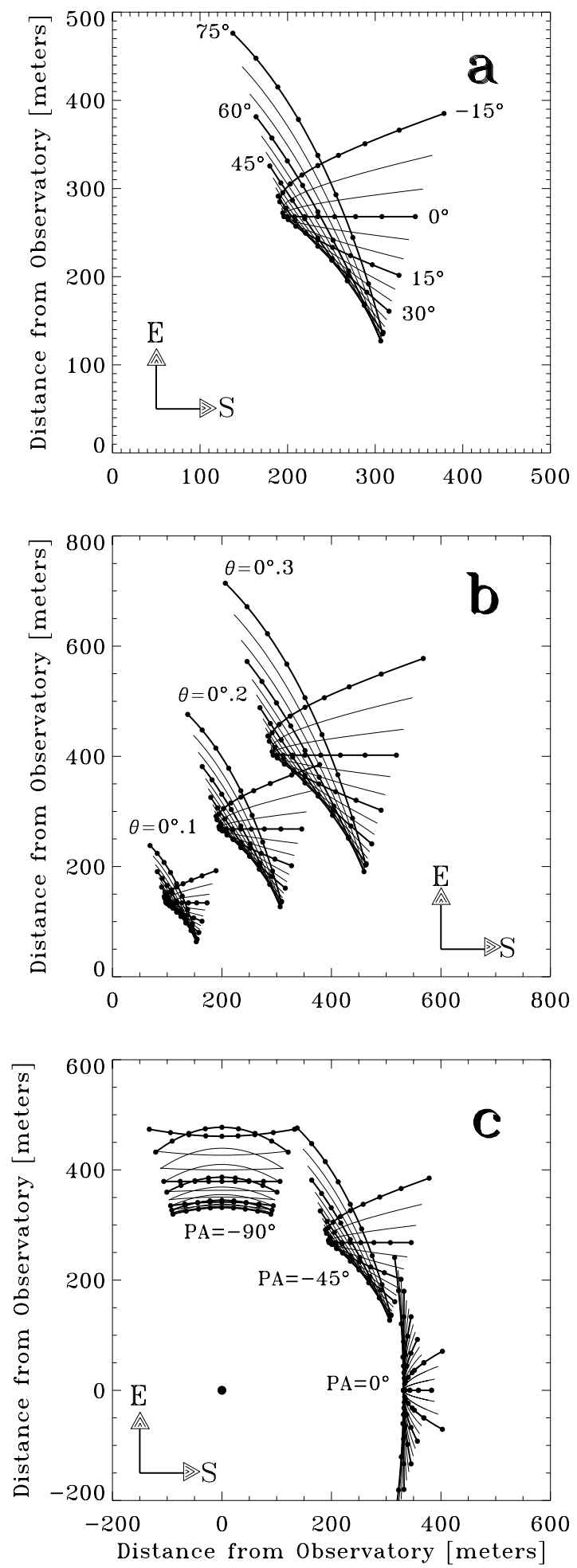

Fig. 4. Paths of auxilary telescope for different values of $\delta, \mathrm{PA}$ and $\theta$. In all cases the observatory latitude is $29^{\circ} \mathrm{N}$. In a) is shown an example with different declinations and fixed position angle and separation. In b) also the separation is varying while in c) this last is kept fixed and position angle varies. For further details see the text

\section{Ground occupation}

The capability of the technique to recover the absolute tilt of the LGS is related to the joint probability to find two stars, whose magnitude is equal or brighter than the limiting magnitude defined in the second section, located at angular distances respectively $d_{1}$ and $d_{2}$ from the observed object as small as possible and forming with respect to the latter an angle of about $90^{\circ}$, in order to reduce to a minimum the error on the tilt determination.

Projecting the problem to the Earth surface, we have the joint probability to exploit two tracks for the small telescopes as close as possible to the main observatory. The area in the neighbourhood of the observatory will have different occupation frequency densities for the auxiliary telescopes depending upon the ratio between the main and the auxiliary telescope diameters $D / D_{\mathrm{a}}$, the Fried parameter $r_{0}$, the tolerance angle $\Delta \psi$ around the nominal position of $90^{\circ}$ and the coordinates of the object.

Using the values given in Sect. 2, several simulations about the ground occupation for $\phi=29^{\circ} \mathrm{N}$ have been performed. For a density of $n=36 \mathrm{deg}^{-2}$ the mean distance of the star nearest to the object $<d_{1}>$ and of the nearest second star $\left\langle d_{2}\right\rangle$, within different tolerance angles (view as a cone of aperture $\Delta \psi$ centered around the $\pm 90^{\circ}$ position) have been computed averaging over 5000 simulation runs.

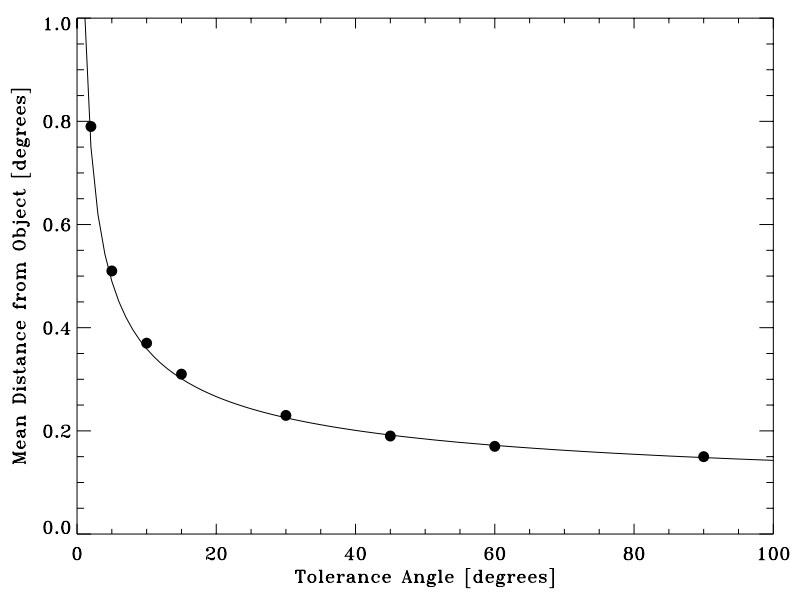

Fig. 5. Mean distance from the observed object of the nearest second star versus the tolerance angle $\Delta \psi$ : the filled circles calculated through the simulations are well fitted by the $-1 / 2$-law of Eq. (14) and the asymptotic trend gives a value of $\approx 0.12$

The mean distance of the nearest star $<d_{1}>$ depends only upon the star density $n$, and its value is equal to:

$<d_{1}>\approx \frac{1}{2 \sqrt{n}}$

In our case a figure of $<d_{1}>\approx 5$ arcmin is obtained. 
The computation of the second nearest star is more complicated because of the constraint imposed by the first nearest star. Using the observation that the square of the mean distance is proportional to the available number of stars in a given sky region one can figure out the following relationship:

$$
<d_{2}>\approx \frac{a}{\sqrt{n \Delta \psi}}+b
$$

where the fixed addictive term is due to take into account the finite value of $\left\langle d_{2}\right\rangle$ when $\Delta \psi$ approaches $180^{\circ}$. Numerical simulations and fitting of the obtained data lead to an estimation, for our case study, of $a \approx 6.0 \mathrm{deg}^{1 / 2}$ and $b \approx 4.310^{-2} \mathrm{deg}$. The results are plotted in Fig. 5 .

In order to define the tracks distribution around the observatory we performed a MonteCarlo simulation in which we calculated the tracks for the star and its almost perpendicular companion closest to the object for different tolerance angles, using a star field of poissonian mean density equal to $n=36 \mathrm{deg}^{-2}$ and objects of random zenithal distance $\leq 30^{\circ}$ considering an observation time of 4 hours across the meridian $\left( \pm 2^{\mathrm{h}}\right)$.

The results for the case $\Delta \psi=45^{\circ}$ are shown in Fig. 6 : the simulated data are rappresented with filled circles and a poissonian fitting is superimposed. The figure shows a poissonian-like distance distribution both for the nearest star and for its perpendicular companion. We performed 5000 simulation runs to figure out the described results.

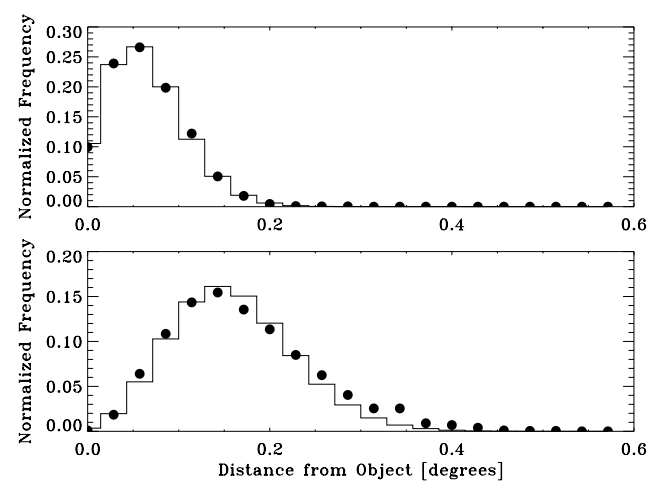

Fig. 6. Distribution of the tracks around the observatory: the MonteCarlo data results are well fitted by a Poissonian curve. The upper plot, referred to the nearest star, shows a sharper shape with respect to the case of the perpendicular companion (lower plot)

A simple view of the MonteCarlo simulation with only 200 tracks is shown in Fig. 7: the tracks of the perpendicular companion are marked at their ends with filled circles. The different distances of the paths for the two reference stars are evident.
Given a circular area of radius $R$ around the observatory it is possible to retrieve the percentage of tracks inside it, and to define the sky coverage of the system. The results are shown in Table 1 for different tolerance angles where the other parameters are the same used in this section; accepting a further degradation of the measurement error due to the non perpendicularity of the two reference stars $\left(29 \%, 48 \%\right.$ and $73 \%$ respectively for $\Delta \psi=60^{\circ}, 75^{\circ}$ and $90^{\circ}$ ), it is possible, within $1 \mathrm{~km}$, to project the LGS through the auxiliary telescopes and track them for approximately all the reference stars found in proximity of the observed object.

Table 1. Percentages of stars tracked related to distance from observatory and to tolerance angle

\begin{tabular}{c|cccccc}
\hline \hline$\Delta \psi$ & $15^{\circ}$ & $30^{\circ}$ & $45^{\circ}$ & $60^{\circ}$ & $75^{\circ}$ & $90^{\circ}$ \\
\hline$R=\mathbf{2 5 0} \mathbf{~ m}$ & 1 & 2 & 3 & 5 & 6 & 7 \\
$R=\mathbf{5 0 0} \mathbf{~ m}$ & 12 & 21 & 29 & 37 & 40 & 48 \\
$R=\mathbf{7 5 0} \mathbf{~ m}$ & 30 & 51 & 65 & 76 & 81 & 86 \\
$R=\mathbf{1 0 0 0} \mathbf{~ m}$ & 51 & 75 & 88 & 94 & 96 & 98 \\
\hline \hline
\end{tabular}

The ground tracking speed is very small. We have found mean values of about few meters per minute: for example, in the condition $\Delta \psi=45^{\circ}$ and maximum zenithal distance of $30^{\circ}$, the mean tracking speed is $\approx 0.7 \mathrm{~m} \mathrm{~min}^{-1}$ for the $1^{\text {st }}$ star and $\approx 1.6 \mathrm{~m} \mathrm{~min}^{-1}$ for its perpendicular companion.

It is worth noting that all calculations are performed for a star density given at the North Galactic Pole and in most cases $n$ is much more greater.

\section{Conclusions}

We analyzed in detail the theoretical problem of ground tracking for auxiliary telescopes in the REM95 technique. We have given a limiting magnitude for the system, we have defined the error due to non perpendicular configuration of the two reference stars and we have shown the geometry of the problem with some track path examples.

We performed several calculations in a particular case study showing the tolerance angle dependence of the mean distance of the perpendicular companion of the closest star and the Poissonian distribution of these two around the observed astronomical target.

It is to be pointed out that we performed such a simulation run assuming the worst case both for the star density and the for requirements to be imposed on the goodness of the correction. Moreover the calculation is made assuming that the objects have to be LGS tilt corrected at any time.

Given a certain ground occupation around the main observatory it can be easily figured out that most objects can be tracked at some particular instant. 


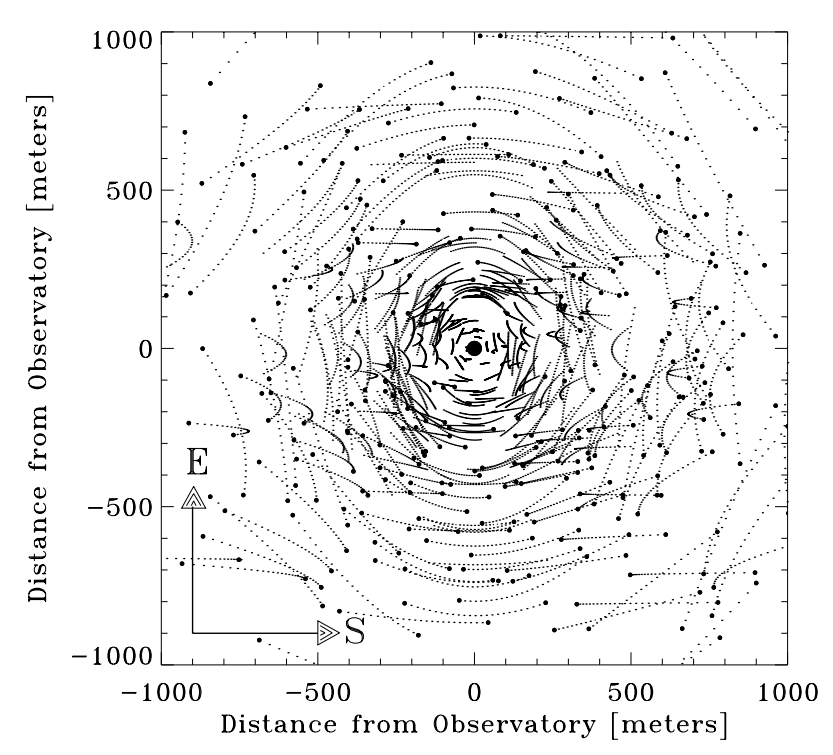

Fig. 7. Example of paths of 200 stars around the observatory (latitude $29^{\circ} \mathrm{N}$ ) for $\Delta \psi=45^{\circ}$, maximum zenithal distance of $30^{\circ}$. The paths of second star are marked at the ends with filled circles

Finally we did not make any attempt to exploit the non-vanishing length of the Sodium beacon as seen from the auxiliary telescopes. Assuming a ratio between the thickness of the Sodium layer with respect to its altitude of the order of ten, at a distance of $500 \mathrm{~m}$ from the main observatory this will translates into a tolerance band of $50 \mathrm{~m}$ width. Non straight paths, with deviations smaller than this size, can be substituted by a straight track.

\section{References}

Bahcall J.N., Soneira R.M., 1981, ApJ 246, 122

Barbieri C., 1996, SPIE Proc. 2871, 244

Belen'kii M.S., 1994, SPIE Proc. 2201, 321

Belen'kii M.S., 1995, SPIE Proc. 2471, 289

Foy R., Boucher Y., Fleury B., Greenberg G., McCullough P.R., Migus A., Tallon M., 1992, ESO Conf. Prof. 42, 437

Foy R., Migus A., Biraben F., Grynberg G., McCullough P.R., Tallon M., 1995, A\&AS 111, 569

Happer W., MacDonald G.J., Max C.E., Dyson E.J., 1994, JOSA-A 11, 263

Lukin I.P., 1996, OSA Technical Digest 13, Addendum AMB35

Olivier S.S., Max C.E., Gavel D.T., Brase J.M., 1993, ApJ 407, 428

Olivier S.S., Gavel D.T., 1994, JOSA-A 11, 368

Pilkington J.D.H., 1987, Nat 330, 116

Ragazzoni R., 1996a, A\&A 305, L13

Ragazzoni R., 1996b, ApJ 465, L73

Ragazzoni R., Esposito S., Marchetti E., 1995, MNRAS 276, L76

Ragazzoni R., Marchetti E., 1996, SPIE Proc. 2871, 948

Rigaut F., Gendron E., 1992, A\&A 261, 677

Tyler G.A., Fried D.L., 1982, JOSA 72, 804

Vivekanand M., Morris D., Downes D., 1988, A\&A 203, 195

Zombeck, M.V., 1990, Handbook of Space Astronomy \& Astrophysics. Cambridge Univ. Press 